\title{
¡Leer, comentar, compartir! El fomento de la lectura y las tecnologías sociales
}

\author{
Read, comment, share! Promoting reading \\ and social technologies
}

Ramón-Alberto MANSO-RODRÍGUEZ1

\section{Resumen}

Se reflexiona sobre los desafíos que suponen los avances tecnológicos para el sector bibliotecario, fundamentalmente los asociados a la llamada Web social y las potencialidades de éstas, para implementar acciones que contribuyan a fomentar y promover el gusto por la lectura en las nuevas generaciones de lectores. De esta forma, en un primer momento, el estudio analiza las tendencias actuales, en términos de comportamiento de los lectores y las herramientas tecnológicas más empleadas para establecer conexiones entre bibliotecarios, autores y lectores, con la finalidad de convertir la lectura en una actividad social. Seguidamente en la presentación se analizan una serie de iniciativas que actualmente se desarrollan en bibliotecas públicas y otras instituciones, para develar elementos a considerar como buenas prácticas en este sentido y con los cuales implementar desde la biblioteca acciones de fomento de la lectura, empleando las herramientas tecnológicas disponibles. Como resultado del estudio se identifican las principales funcionalidades que ofrecen los clubes de lectura en el entorno de Internet, como acciónfundamental para la promoción de la lectura. De igual forma se observó cómo dichas actividades empleen como tecnologías para su implementación, los blog y las redes sociales y en menor medida los canales para el intercambio de archivos y los Wiki. Finalmente se ofrecen recomendaciones para el desarrollo de esta modalidad de servicio en cualquier biblioteca.

Palabras ilave: Bibliotecas públicas del Estado. Fomento de la lectura. Tecnologías sociales. Usuarios. Web 2.0.

\begin{abstract}
We reflect on the challenges posed by technological advances for the library sector, primarily those associated with the so-called social Web and their potential to implement actions that help foster and promote a love for reading in new generations of readers. Thus, at first, we analyzed the current trends in terms of reader behavior and technological tools used to establish connections between librarians, authors and readers, in order to make reading a social activity. Next, we examine a number of initiatives currently being developed at public libraries and other institutions to unveil elements considered as good practices and actions that promote reading using the technological tools available. As a result of the study, we identified the main features offered by book clubs in the Internet environment as fundamental to the promotion of reading. Similarly we observed how these activities use technologies, blogs and social networks and, at a lower rate, file sharing and Wiki. Finally, we recommend the development of this type of service at any library.
\end{abstract}

Keywords: Public libraries. Promoting reading. Social technologies. Users. Web 2.0.

\section{Introducción}

En la actualidad el debate entorno al fomento de lectura debe asentarse sobre la base de tres pilares fundamentales: las necesidades y preferencias de una nueva generación de lectores, la incidencia del uso prolífero de innumerables dispositivos electrónicos por parte de estos y los nuevos medios y formas de lecturas.

\footnotetext{
1 Universidad Central Marta Abreu de Las Villas, Centro de Estudios de Educación, Facultad de Ciencias de la Información y la Educación. Carretera a Camajuaní, km5 y 1/2, Santa Clara, Cuba.E-mail:<manso@uclv.edu.cu>.

Recebido el 5/9/2013, re-presentado el 6/3/2014 y aceptado para su publicación el 28/3/2014.
} 
Todo ello propicia que la biblioteca tenga ante sí un gran reto ¿cómo fomentar y promover el gusto por la lectura en las nuevas generaciones de lectores? Desafío que en primer término debe partir precisamente del reconocimiento de las necesidades y preferencias de los lectores, algo que puede resultar complejo, dado en parte por la diversidad de segmentos etáreos que se atienden desde la propia institución.

Por otro lado se vive una época en que la tecnología inunda cada espacio en el que habitamos y la irrupción y auge de los medios audiovisuales han propiciado un cambio en el modelo cultural, pasando de la supremacía de una cultura alfabética, textual e impresa a un modelo construido a base de imágenes audiovisuales.

Es así como el placer de leer un libro, ha cedido espacio ante medios como la televisión, Internet, juegos interactivos, entre otros, los que hoy centran la atención e interés de las jóvenes generaciones. Influye de igual forma la manera en que estos jóvenes crean, gestionan y consumen la información en la actualidad.

Aparejado a la diversificación de soportes, las tecnologías disponibles favorecen que el lector se enfrente a nuevas formas de lectura. La aparición del hipertexto ha dado paso de una lectura lineal, a una multisecuencial y multilineal, que permite leer rastreando, o sea, saltándose párrafos o bloques de información, así como la posibilidad, a través de enlaces, de abrirse a nuevas fuentes de información que complementan la principal.

Se observa entonces que el entorno donde los lectores interactúan a diario se ve influenciado por las diferentes herramientas tecnológicas y dispositivos existentes, el cambio hacia una cultura más visual que textual y por los nuevos soportes y formas de lectura. Elementos que las bibliotecas deben reconocer y aprovechar para construir un escenario más adecuado a las necesidades e intereses de estos grupos de lectores y afianzar en ellos los hábitos de la lectura.

El análisis de estos elementos resulta indispensable para el desarrollo e implementación de proyectos socialmente interactivos, donde a través de una participación activa del lector, se logre que la lectura resulte significativa para esta generación.
En este sentido son innegables los esfuerzos que desde la biblioteca se realizan para lograr que la lectura sea valorada por la sociedad y particularmente por las generaciones más jóvenes. Muchas de estas iniciativas hacen un uso intensivo de las tecnologías sociales (blogs, wikis, sitios de redes sociales, etc.), constituyendo estos medios, mecanismos idóneos y eficaces para complementar la labor de fomento en el uso de los libros y el gusto por la lectura que se realiza en las bibliotecas (Celaya, 2007; Manso-Rodríguez, 2012).

\section{Métodos}

El estudio que se presenta tiene un alcance descriptivo. El tipo de investigación descriptiva, busca especificar propiedades, características y rasgos importantes de cualquier fenómeno que se analice. Resultando de utilidad para mostrar con precisión los ángulos o dimensiones de un fenómeno, suceso, comunidad, contexto o situación. Los estudios descriptivos miden conceptos o variables para decir cómo se comporta el fenómeno de interés, sin establecer relaciones entre las variables medidas (Hernández Sampieri et al., 2006).

Dado su alcance, para la realización de la investigación se emplearon los métodos empíricos del análisis documental clásico y los estudios de caso, los cuales permitieron en un primer acercamiento, a través de la literatura referente al tema, detallar las diferentes herramientas y potencialidades de las mismas para ofrecer las acciones para el fomento de la lectura. Con posterioridad, se observó un conjunto de servicios que en este sentido se ofrecen en la Web y a partir de su estudio especificar propiedades, características y rasgos importantes, con los cuales establecer los elementos que todo servicio de fomento de la lectura, que emplee tecnologías en su desarrollo, debe observar para su implementación.

La muestra la conformaron 14 proyectos (Cuadro 1), tanto de habla hispana como anglosajona, de los cuales siete son desarrollados por bibliotecas públicas, correspondiendo el resto a otras organizaciones interesadas en el fomento del hábito de la lectura. Para la selección de dicha muestra se realizó una búsqueda global en Internet, tomándose aleatoriamente las experiencias, algunas de las cuales resultaban 
Cuadro 1. Proyectos de fomento de la lectura a evaluar. 2014.

\begin{tabular}{|c|c|}
\hline Proyecto & Dirección electrónica \\
\hline Folec, portal para el fomento de la lectura & <http://www.fomentolectura.es/> \\
\hline Lecturalia & $<$ http://www.lecturalia.com/> \\
\hline aNobii & $<$ http://www.anobii.com/> \\
\hline Book country & $<$ http://bookcountry.com/> \\
\hline Entrelectores & <http://www.entrelectores.com/> \\
\hline Goodreads & <http://www.goodreads.com> \\
\hline Quelibroleo.com & <http://www.quelibroleo.com/> \\
\hline Club de Lectura Virtual, Biblioteca de Barcelona & $<$ http://www.clubdelectura.net> \\
\hline Ciberclub de Lectura, Bibliotecas Municipales de A Coruña & <http://ciberclublectura.wordpress.com/> \\
\hline Club de Lectura El grito, Biblioteca Pública de Albacete & <http://clubelgrito.blogspot.com/> \\
\hline Book Buzz: Virtual Book Club, Toronto Public Library & <http://www.torontopubliclibrary.ca/bookbuzz/> \\
\hline Online Book Club, San José Public Library & <http://www.sjpl.org/tags/online-book-club> \\
\hline Online Book Club, Haliburton County Public Library's & <http://www.goodreads.com/group/show/82817-hcpl-s-online-book-club> \\
\hline Online Book Club: Daily Book Samples, Bibliotecas Públicas EE.UU & <http://www.dearreader.com/> \\
\hline
\end{tabular}

Fuente: Elaboración propia (2014).

Nota: EE.UU: Estados Unidos.

representativas, dado su referencia en la literatura analizada (Cruz González-Cutre \& Saurin Parra, 2008; Seoane, 2010; Manso-Rodríguez, 2012).

Para la valoración de cada uno de estos proyectos y observar su funcionamiento, con vistas a identificar elementos comunes y diferencias más representativas, resultó necesario normalizar los datos sobre cada página. En este sentido se elaboró una guía, que contempló los siguientes elementos: denominación, modalidad, público al que va dirigido, cantidad de usuarios, alcance temático, entidad organizadora, tecnologías que usan y funcionalidades o servicios que ofrece.

Como modalidad de actividad para el fomento del hábito de lectura, se determinó valorar la implementación del Club de Lectura, por ser ésta la acción más común entre las instituciones bibliotecarias y de otras organizaciones que ofrecen a su comunidad usuaria, una vía para la promoción del libro y la lectura.

\section{El lector, el fomento de la lectura y la Web social}

Hoy en día, las acciones que la biblioteca realice para desarrollar o reforzar el hábito de la lectura, deben lograr despertar interés y erigirse como espacios de expresión y comunicación en los que el lector encuentre una amplia oferta cultural, contribuyendo de esta manera, a su formación como lectores competentes, críticos y capaces de dominar las diferentes formas de lecturas presentes en la actualidad y de discriminar la abundante información a la que tienen acceso (Abello, 2010; Lozano, 2010).

Para lograr estos fines es primordial definir la tipología de lectores a atender, elemento que permitirá identificar los gustos, preferencias y hábitos de lectura de la comunidad de lectores asociados a la biblioteca. De manera general, en estos tiempos, se pueden reconocer y definir tres tipos de lectores: el tradicional, el polivalente y el digital.

El lector tradicional siente cierta reticencia por los medios digitales para la lectura, necesita aún hojear el libro y percibir el olor característico del título recién salido de imprenta, en cambio, al otro extremo se encuentra el lector digital, quien es un gran consumidor de las tecnologías, concibe su mundo alrededor de las redes, leyendo y comunicándose solo a través de ella y rara vez a tomado un libro en sus manos, y en término medio, el polivalente, quien tiene aptitudes para utilizar diferentes formas de lectura, para apropiarse de diferentes tipos de textos y escritos y es capaz de leer en diferentes soportes.

Por lo general, dentro de las categorías de lector polivalente y del digital, podemos englobar a las 
generaciones más jóvenes. Estos lectores se caracterizan, entre otros elementos, por poseer una amplia cultura digital, y por ello están acostumbrados a utilizar disímiles tecnologías y a compartir sus gustos, preferencias y hasta contenidos, en espacios de socialización.

Al ser estos los segmentos etáreos a los que se debe encaminar los esfuerzos para fomentar y afianzar los hábitos de la lectura, requerirá la construcción de un escenario más adecuado a sus necesidades e intereses. Una vía para ello, es aprovechando las potencialidades que los avances tecnológicos ofrecen y propiciar así espacios donde la comunidad se "reúna" con dicha finalidad.

Es así, como las bibliotecas para ofrecer las actividades de fomento de la lectura, deben apostar por la innovación, que basada en la cooperación, la búsqueda de sinergia y el uso eficiente de los recursos, permita la presentación de las mismas, de forma diferente, diversificada, atractiva e impregnada de la filosofía 2.0 (Celaya, 2007; Lozano, 2010).

Por tanto se han de encaminar los esfuerzos para crear espacios, que desde la biblioteca, contribuyan a conectar a los lectores entre ellos y con los libros que les son afines, en el momento adecuado y el lugar correcto (Abram, 2008), así como propiciar el acercamiento entre lectores, autores y bibliotecarios, todos en un ambiente colaborativo y con una participación activa de cada uno de ellos.

Sin lugar a dudas, aprovechando las tecnologías sociales, los lectores han encontrado un mecanismo donde expresarse sin ningún tipo de intermediación, por tal motivo estas se han convertido en la vía idónea para obtener información y compartir opiniones sobre libros y autores, actuando como fuentes complementaria a las oficiales. Veamos algunas de las potencialidades de algunas de las herramientas más utilizadas en el desarrollo de acciones para el fomento de la lectura.

Blog: Uno de los usos más frecuente de este tipo de tecnología, para la implementación de acciones de fomento de la lectura, es para el desarrollo de un Club de Lectura Virtual. Entre las ventajas que se le reconocen a los blog para el desarrollo de estas acciones, Seoane (2010, online), plantea como principales:

- Permiten crear una comunidad de lectores con los mismos hábitos, gustos, preferencias, etc. y su participación.
- Favorece la creación de una fuente de información complementaria a los suplementos y revistas culturales tradicionales.

- Es una vía fiable de intercambio de información sobre libros entre lectores.

- Motivan la creación a partir de más visibilidad a lo escrito y lo publicado.

- Constituyen una forma de comunicación bidireccional.

- No requiere desembolso económico.

- Su actualización es sencilla y constante.

- Posibilidades de vincularse con otros blog o contenidos.

- Facilita la organización cronológica de eventos.

Con relación a esta tecnología, Richardson (2006) señala que al ser una herramienta que se emplea para el intercambio de ideas y como un recurso para interconexión y comentarios sobre las ideas relacionadas entre sí, son una vía idónea para promover el pensamiento crítico, analítico, creativo, intuitivo, asociativo y analógico, además para potenciar el acceso a la información de calidad y combinar la interacción de lo individual con lo social.

Wiki: La utilización de los Wikis, para el desarrollo de acciones para el fomento de la lectura, según Celaya (2009), aporta una serie de valores que se deben tener en cuenta, entre ellos: favorece el desarrollo de la capacidad de argumentación para hacer valor un punto de vista frente a otros, contrastar información de diferentes fuentes, valorar la relevancia de los datos encontrados y reconocer el valor de los textos firmados frente a los anónimos. Este autor además enuncia, que independientemente de las imperfecciones que en esta plataforma se puedan observar, la misma ofrece la posibilidad de mostrar a los jóvenes lectores la complejidad de la elaboración de contenidos, los diferentes puntos de vista que puede tener una historia y la importancia de contrastar las fuentes, por citar algunas potencialidades.

Entre las utilidades principales de esta herramienta están: la creación de biografías de autores, materiales de consulta y apoyo a las disímiles actividades y para el desarrollo de concursos literarios. También resulta atractiva para la elaboración de antologías, con 
la selección, organización y presentación de textos según determinados criterios y la recopilación y presentación de fuentes documentales diversas, como por ejemplo: artículos de prensa, reseñas de libros y cuentos o poemas escritos por los propios usuarios, entre otras opciones creativas e innovadoras (Arreguin, 2004; Chawner \& Lewis, 2006; Seoane, 2010).

Sitios de Redes Sociales: Con respecto al empleo de los sitios de redes sociales en el fomento de la lectura, estas herramientas ofrecen la posibilidad de mantener un flujo de comunicación constante con los lectores y estos a su vez con otros, beneficiándose ambas partes, pues al conocer mediante la recomendación de libros entre estos últimos, la biblioteca podrá monitorear sus gustos, necesidades e intereses (Manso-Rodríguez, 2012).

Los sitios de redes sociales además favorecen la creación de espacios de lectura y escritura más cercanos a la manera en que los lectores se comunican actualmente, redundando en una mayor motivación por el placer de leer (Celaya, 2009). Siendo sus usos más frecuentes, para la implementación de Club de Lectura Virtual y como vía para intercambiar archivos multimedia.

Intercambio de archivos y datos: Otra alternativa, con relación al fomento de la lectura, que las bibliotecas deben evaluar, son las diferentes plataformas o herramientas para el intercambio de archivos audiovisuales y datos, entre ellas por ejemplo: YouTube, Flickr, Slideshare, Really Simple Syndication (RSS).

El empleo desde la biblioteca, de algunas de estas herramientas, permite desarrollar un conjunto de acciones para el fomento de la lectura, actividades que según algunos autores (Bolan et al., 2007; Luo, 2009) comprenderán de manera general la realización de:

- videos con entrevista a autores o de actividades de promoción desarrollada en la biblioteca.

- fotos relacionadas con los encuentros de escritores o la presentación de libros la digitalización de la cubierta o algunas páginas de los libros.

- grabación de fragmentos de libros o entrevistas con autores en formato de audio.

- la elaboración de presentaciones, orientaciones bibliográficas, guías de lectura y tutoriales (sobre cómo utilizar el catálogo, ilustrar el contenido del curso de alfabetización informacional, sobre la búsqueda, recuperación y evaluación de la información, cómo acceder y utilizar bases de datos de la biblioteca, etc...).

Acciones que se pueden complementar, según sugiere Celaya (2009), con la participación activa del lector en los procesos de creación, etiquetado y publicación en la red.

Aprovechando estas funcionalidades, la biblioteca, puede crear su propio perfil o canal y subir o destacar como favoritos en su propio espacio archivos audiovisuales, además elaborarlos en el centro, grabando las actividades culturales y de animación a la lectura que llevan a cabo, para posteriormente colgarlas en la Red, acción que puede ser auxiliada por los propios usuarios.

Arya y Mishra (2012) y Buigues-García y GiménezChornet (2012), señalan en relación con la utilización de los RSS, la disponibilidad de la información en cualquier interfaz, las posibilidades para descubrir nuevas fuentes de interés: notificaciones de nuevos resultados de búsquedas, artículos publicados, fotos, etc.; la privacidad al evitar el uso del correo electrónico para subscribirse a las fuentes y la protección contra el spam y los virus.

Resulta evidente, que para intentar motivar en las nuevas generaciones los hábitos de la lectura, y que esta se convierta en una opción de ocio, se debe procurar emplear algunas de las herramientas descritas con anterioridad. Al hacer una mirada en la Web, encontramos múltiples iniciativas en este sentido, el análisis de algunas de ellas, dará las pistas de los elementos a considerar para desarrollar esta modalidad de servicio desde cualquier biblioteca que desee implementar su club de lectura en ambiente virtual.

\section{Algunas realidades, el fomento de la lectura en la Web social}

Del análisis anterior observamos que una de las potencialidades que ofrecen el empleo de las tecnologías sociales para propiciar el desarrollo de acciones para el fomento de la lectura, es la implementación de los Clubs de Lectura, actividad que tradicionalmente la biblioteca ofrece a su comunidad de usuarios y que es considerado como un grupo informal de lectores que se reúnen para leer un mismo libro y posteriormente comentarlo y discutir sus puntos de vista entre todos (Reitz, 2010). 
Así podemos definir como un Club de Lectura Virtual, al espacio de encuentro, que basado en el empleo de herramientas tecnológicas, propicia que los lectores se "reúnan" para exponer sus criterios y puntos de vista sobre determinada lectura, debatir, valorar y sugerir obras literarias.

Señalan Cruz González-Cutre y Saurin Parra (2008), que existen dos modalidades: los que fijan un día y hora para la discusión, de un modo similar al típico chat, y en otros la participación es atemporal: los participantes dejan sus opiniones en cualquier momento. De igual manera pueden ser considerados como: generales cuando su finalidad es promover cualquier temática o dedicados a un interés o género específico, si se organizan con dicha finalidad. Si por el contrario tenemos en cuenta quienes lo organizan, estos pueden ser institucionales, cuando son promovidos por bibliotecas, librerías, editoriales $u$ otras organizaciones asociadas al mundo del libro y la literatura y personales, si son creados por un escritor o lector interesado en crear su propio espacio de promoción.

En efecto, un club de lectura virtual, es ya una realidad para muchas organizaciones, entre las que por supuesto se incluyen las bibliotecas, pero ¿cuáles son las funcionalidades o servicios más comunes? ¿qué patrón seguir para desarrollar eficientemente esta modalidad de servicio? Una valoración de algunas de estas experiencias permitirá evacuar estas interrogantes y de este modo adquirir ideas que permitan un mejor desarrollo de esta actividad. Aspectos que primeramente son abordados de manera particular por cada proyecto y posteriormente analizados en su conjunto.

En las Cuadro 2 y Cuadro 3 se muestra la información general recopilada de cada uno de los proyectos. Para su análisis han sido divididos en dos grupos bajo el criterio de la entidad que lo organiza, ya sean instituciones bibliotecarias o perteneciente a otros organismos o asociaciones.

Por regla general todos los servicios evaluados se centran en la promoción del libro para su consulta y diseminación por parte de los usuarios que consultan el sitio, enfocados fundamentalmente en promover los valores de determinada obra, divulgar las características del libro que puedan ser atractivas para los usuarios y brindar datos de los autores e informaciones diversas sobre el mundo del libro y la literatura. En resumen se puede observar que entre las funcionalidades más comunes, se encuentran: Crear y/o unirse a grupos de interés; Publicar sugerencias de libros; Publicar comentarios sobre un libro; Valorar mediante sistema de puntos los libros; Foro/Chat para contacto de la comunidad; Encuentro con escritores; Publicar fotos/ actividades/biografías de autores; Guías de Lectura; Debate guiado por preguntas; Presencia en los sitios de redes sociales; Enlaces a la crítica especializada; Crear lista de autores/libros preferidos; Información general del Club.

Aunque no existen muchas diferencias entre las funcionalidades que brindan los servicios regentados por bibliotecas y los ofrecidos por librerías u otras organizaciones, de manera particular se presentan elementos distintivos que son por tanto característicos de cada uno y a la vez diferenciadores.

La mayor diferencia reside en las fuentes documentales que se proponen para la lectura, en las instituciones bibliotecarias éstas proceden en gran medida de sus fondos y el objetivo del club es precisamente promover la circulación de las obras que se atesoran en la biblioteca y por tanto están condicionadas, aparte de los intereses del usuario, por la disponibilidad del título dentro de la institución. En cambio en las iniciativas que responden a otras instituciones relacionadas con el mundo del libro, lo primero es promover la obra literaria, en algunos casos con un marcado interés comercial, aunque la gran mayoría lo que persigue es fomentar la lectura basada en los intereses consensuados de los miembros del club.

Se observa de manera general, que los proyectos analizados emplean como tecnología para implementar el servicio, los blog y las redes sociales (principalmente: Facebooky Twitter) y en menor medida emplean los Wikis y los sitios para el intercambio de archivos, resultando YouTube y los canales RSS, los más comunes.

Valorando cada característica general o específica de los proyectos analizados, para desarrollar acciones de fomento de la lectura potenciadas desde la biblioteca y empleando herramientas de la Web social, se sugiere seguir un conjunto de pasos, modificados a partir de la propuesta de Manso-Rodríguez (2012) y entre los que se pueden mencionar: 
Cuadro 2. Información general de los proyectos desarrollados por instituciones no bibliotecarias (2014).

\begin{tabular}{|c|c|c|c|c|c|c|}
\hline Denominación & \multicolumn{2}{|l|}{ Folec } & \multicolumn{2}{|l|}{ Lecturalia } & aNobii & Book country \\
\hline Modalidad & \multicolumn{2}{|l|}{ Asincrónico } & \multicolumn{2}{|l|}{ Asincrónico } & Asincrónico & Asincrónico \\
\hline $\begin{array}{l}\text { Público al que va } \\
\text { dirigido }\end{array}$ & \multicolumn{2}{|c|}{$\begin{array}{l}\text { Adolescentes (12 a } 18 \\
\text { años) }\end{array}$} & \multicolumn{2}{|l|}{ Público general } & Público general & $\begin{array}{l}\text { Público general (más de } 18 \\
\text { años) }\end{array}$ \\
\hline Usuarios & \multicolumn{2}{|l|}{55} & \multicolumn{2}{|l|}{64.083} & 68.154 & 1.166 \\
\hline Alcance temático & \multicolumn{2}{|c|}{ Literatura infantil } & \multicolumn{2}{|l|}{ Literatura general } & Literatura general & $\begin{array}{l}\text { Romance, fantasía, misterio, } \\
\text { ciencia ficción }\end{array}$ \\
\hline $\begin{array}{l}\text { Entidad } \\
\text { organizadora }\end{array}$ & \multicolumn{2}{|l|}{ e-Infosfera } & \multicolumn{2}{|l|}{ Ontecnia } & HMV Group & Penguin Group, Pearson \\
\hline $\begin{array}{l}\text { Tecnologías que } \\
\text { usan }\end{array}$ & \multicolumn{2}{|c|}{$\begin{array}{l}\text { Blog, Wiki, Redes Sociales y } \\
\text { Canal YouTube }\end{array}$} & \multicolumn{2}{|c|}{$\begin{array}{l}\text { Blog, Redes Sociales y } \\
\text { Canal RSS }\end{array}$} & Blog y Redes Sociales & Blog y Redes Sociales \\
\hline $\begin{array}{l}\text { Funcionalidades } \\
\text { o servicios que } \\
\text { ofrece }\end{array}$ & \multicolumn{2}{|c|}{$\begin{array}{l}\text { Noticias relacionadas con } \\
\text { la lectura, Tutoriales para } \\
\text { el desarrollo de habilidades } \\
\text { lecto-escritoras, Ranking } \\
\text { de libros por más votados } \\
\text { o comentados, Wiki para } \\
\text { colgar relatos, suscripción } \\
\text { a canal RSS, lista de libros } \\
\text { favoritos, estimulación a } \\
\text { usuarios por participar. }\end{array}$} & \multicolumn{2}{|c|}{$\begin{array}{l}\text { Introducir comentarios, } \\
\text { puntear libros, añadir o } \\
\text { modificar fichas de libros } \\
\text { y autores, información } \\
\text { sobre novedades, enlace } \\
\text { a artículos de opinión } \\
\text { especializada, ofrece } \\
\text { recomendaciones, } \\
\text { suscripción a canal RSS. }\end{array}$} & $\begin{array}{l}\text { Crear y administrar una } \\
\text { biblioteca virtual, } \\
\text { categorizar libros leídos, } \\
\text { crear comunidad de } \\
\text { lectores afines, enviar } \\
\text { mensajes privados, recibir } \\
\text { sugerencias, crear reseñas } \\
\text { de libros, comentar o } \\
\text { puntear los libros, incluir } \\
\text { widget, versión para } \\
\text { móviles, obtener criticas e } \\
\text { información bibliográfica } \\
\text { mediante el escaneo de } \\
\text { código de barra del libro. }\end{array}$ & $\begin{array}{l}\text { Los autores pueden publicar } \\
\text { una obra o parte de ella, } \\
\text { introducir críticas, } \\
\text { comentarios o sugerencias, } \\
\text { recibir información } \\
\text { actualizada sobre las } \\
\text { actividades del sitio, estimular } \\
\text { la participación de sus } \\
\text { miembros. }\end{array}$ \\
\hline \multicolumn{2}{|l|}{ Denominación } & \multicolumn{2}{|l|}{ Entrelectores } & \multicolumn{2}{|c|}{ Goodreads } & Quelibroleo.com \\
\hline \multicolumn{2}{|l|}{ Modalidad } & \multicolumn{2}{|l|}{ Asincrónico } & \multicolumn{2}{|c|}{ Asincrónico } & Asincrónico \\
\hline \multicolumn{2}{|c|}{ Público al que va dirigido } & \multicolumn{2}{|c|}{ Público general } & \multicolumn{2}{|c|}{ Público general (más de 13 años) } & Público general \\
\hline \multicolumn{2}{|l|}{ Usuarios } & \multicolumn{2}{|l|}{15.745} & \multicolumn{2}{|c|}{16.000 .000} & 59.419 \\
\hline \multicolumn{2}{|l|}{ Alcance temático } & \multicolumn{2}{|c|}{ Literatura general } & \multicolumn{2}{|c|}{ Literatura general } & Literatura general \\
\hline \multicolumn{2}{|c|}{ Entidad organizadora } & \multicolumn{2}{|c|}{$\begin{array}{l}\text { Evoluziona, DosDoce, Nervia, } \\
\text { Ediciona y Gavilán Digital }\end{array}$} & \multicolumn{2}{|c|}{$\begin{array}{l}\text { Goodreads Inc. (sitio personal de } \\
\text { Otis Chandler) }\end{array}$} & Grupo RC S.L. \\
\hline \multicolumn{2}{|c|}{ Tecnologías que usan } & \multicolumn{2}{|c|}{ Blog, Redes Sociales y Canal RSS } & \multicolumn{2}{|c|}{ Blog, Redes Sociales } & $\begin{array}{l}\text { Blog, Redes Sociales y Canal } \\
\text { YouTubey RSS }\end{array}$ \\
\hline \multicolumn{2}{|c|}{$\begin{array}{l}\text { Funcionalidades o servicios que } \\
\text { ofrece }\end{array}$} & $\begin{array}{l}\text { Compartir in } \\
\text { opiniones, cr } \\
\text { lectura, com } \\
\text { miembros, re } \\
\text { recomendac } \\
\text { con autores, } \\
\text { comentar lib } \\
\text { canal RSS, añ } \\
\text { compartirlos }\end{array}$ & $\begin{array}{l}\text { formación, } \\
\text { síticas y gustos de } \\
\text { unicarse entre los } \\
\text { ecibir } \\
\text { iones, conversar } \\
\text { puntear o } \\
\text { ros, suscripción a } \\
\text { ladir libros y }\end{array}$ & $\begin{array}{l}\text { Punte } \\
\text { conec } \\
\text { autor } \\
\text { recom } \\
\text { añadi } \\
\text { de tít }\end{array}$ & $\begin{array}{l}\text { ar y comentar libros, } \\
\text { tar con otros lectores y } \\
\text { s, recibir } \\
\text { endaciones de lectura, } \\
\text { libros, crear estanterías } \\
\text { los. }\end{array}$ & $\begin{array}{l}\text { Selección de la lectura del mes } \\
\text { mediante sistema de votos, hacer } \\
\text { comentarios, canal YouTubeyRSS, } \\
\text { listar las sugerencias por varias } \\
\text { categorías, informarse de eventos } \\
\text { o noticias generales, recibir } \\
\text { recomendaciones, comentar o } \\
\text { puntear los libros, crear biblioteca } \\
\text { personalizada. }\end{array}$ \\
\hline
\end{tabular}

Fuente: Elaboración propia (2014).

Nota: RSS: Really Simple Sindication. 
Cuadro 3. Información general de los proyectos desarrollados por instituciones bibliotecarias (2014).

\begin{tabular}{|c|c|c|c|c|c|c|}
\hline Denominación & \multicolumn{2}{|c|}{ Club de lectura virtual } & \multicolumn{2}{|l|}{ Ciberclub de lectura } & Club de lectura el grito & Book buzz: virtual book club \\
\hline Modalidad & \multicolumn{2}{|c|}{ Asincrónico/Sincrónico } & \multicolumn{2}{|l|}{ Asincrónico } & Asincrónico & Asincrónico/Sincrónico \\
\hline $\begin{array}{l}\text { Público al que va } \\
\text { dirigido }\end{array}$ & \multicolumn{2}{|c|}{ Público general } & \multicolumn{2}{|l|}{ Público general } & Público general & Público general \\
\hline Usuarios & \multicolumn{2}{|l|}{7.000} & \multicolumn{2}{|l|}{10.368} & 403 & 802 \\
\hline Alcance temático & \multicolumn{2}{|c|}{$\begin{array}{l}\text { Novelas autores } \\
\text { contemporáneos }\end{array}$} & \multicolumn{2}{|l|}{ Literatura general } & $\begin{array}{l}\text { Novela negra, Fantasía, } \\
\text { Terror, Policiaco }\end{array}$ & Literatura general \\
\hline $\begin{array}{l}\text { Entidad } \\
\text { organizadora }\end{array}$ & \multicolumn{2}{|c|}{ Biblioteca de Barcelona } & \multicolumn{2}{|c|}{$\begin{array}{l}\text { Bibliotecas Municipales } \\
\text { de A Coruña }\end{array}$} & $\begin{array}{l}\text { Biblioteca Pública de } \\
\text { Albacete }\end{array}$ & Toronto Public Library \\
\hline $\begin{array}{l}\text { Tecnologías que } \\
\text { usan }\end{array}$ & \multicolumn{2}{|c|}{ Blog y Redes Sociales } & \multicolumn{2}{|c|}{$\begin{array}{l}\text { Blog, Redes Sociales y } \\
\text { Canal RSS }\end{array}$} & $\begin{array}{l}\text { Blog, Redes Sociales, } \\
\text { Canal RSS y Podcasts }\end{array}$ & Blog, Redes Sociales y Canal RSS \\
\hline $\begin{array}{l}\text { Funcionalidades o } \\
\text { servicios que ofrece }\end{array}$ & \multicolumn{2}{|c|}{$\begin{array}{l}\text { Comentar el libro } \\
\text { propuesto, charlar con } \\
\text { el autor, presentar } \\
\text { materiales de ayuda } \\
\text { para la lectura, videos } \\
\text { con entrevistas al autor } \\
\text { o escenas de la obra, } \\
\text { enlaces a la crítica } \\
\text { especializada, acceso a } \\
\text { capítulos del libro, } \\
\text { datos del autor y de } \\
\text { sus obras, información } \\
\text { sobre el libro y los } \\
\text { personajes, } \\
\text { recomendaciones de } \\
\text { lectura, foro. }\end{array}$} & \multicolumn{2}{|c|}{$\begin{array}{l}\text { Comentar libros, sugerir } \\
\text { nuevas adquisiciones, } \\
\text { lectura y debate guiado } \\
\text { por plazos y preguntas, } \\
\text { imagen de la portada, } \\
\text { nube de palabras, } \\
\text { efemérides, listado de } \\
\text { títulos más populares, } \\
\text { calendario de plazos } \\
\text { para la lectura, } \\
\text { suscripción a noticias } \\
\text { por correo electrónico y } \\
\text { RSS. }\end{array}$} & $\begin{array}{l}\text { Promocionar } \\
\text { actividades del club, } \\
\text { recomendaciones de } \\
\text { lectura, suscripción a } \\
\text { noticias por correo } \\
\text { electrónico y RSS, } \\
\text { enlace los libros al } \\
\text { catálogo social } \\
\text { LibraryThing, descargar } \\
\text { o escuchar podcasts } \\
\text { de las emisiones del } \\
\text { club en la radio local. }\end{array}$ & $\begin{array}{l}\text { La lectura del mes es promovida a } \\
\text { partir de la sugerencia de los } \\
\text { miembros, permite intercambiar } \\
\text { opiniones, conocer e interactuar con } \\
\text { el escritor, suscripción a noticias por } \\
\text { correo electrónico, debate guiado } \\
\text { por preguntas, reseñas de libros, } \\
\text { concurso anual de mejores lecturas, } \\
\text { suscripción a canal RSS. }\end{array}$ \\
\hline \multicolumn{2}{|l|}{ Denominación } & \multicolumn{2}{|c|}{ Online book club } & \multicolumn{2}{|c|}{ Online book club } & Online book club: daily book samples \\
\hline \multicolumn{2}{|l|}{ Modalidad } & \multicolumn{2}{|l|}{ Asincrónico } & \multicolumn{2}{|c|}{ Asincrónico } & Asincrónico \\
\hline \multicolumn{2}{|c|}{ Público al que va dirigido } & \multicolumn{2}{|c|}{ Público general } & \multicolumn{2}{|c|}{ Público general } & Público general \\
\hline \multicolumn{2}{|l|}{ Usuarios } & \multicolumn{2}{|l|}{2.220} & \multicolumn{2}{|l|}{36} & 375.000 \\
\hline \multicolumn{2}{|l|}{ Alcance temático } & \multicolumn{2}{|c|}{ Literatura general } & \multicolumn{2}{|c|}{ Literatura general } & Ficción, Misterio, Romance, Suspense \\
\hline \multicolumn{2}{|l|}{ Entidad organizadora } & \multicolumn{2}{|c|}{ San José Public Library } & \multicolumn{2}{|c|}{$\begin{array}{l}\text { Haliburton County Public } \\
\text { Library's }\end{array}$} & $\begin{array}{l}\text { Public Library's Public Library's } \\
\text { EE.UU }\end{array}$ \\
\hline \multicolumn{2}{|l|}{ Tecnologías que usan } & \multicolumn{2}{|c|}{ Blog y Redes Sociales } & \multicolumn{2}{|c|}{ Blog y Redes Sociales } & Blog y Redes Sociales \\
\hline \multicolumn{2}{|c|}{$\begin{array}{l}\text { Funcionalidades o servicios } \\
\text { que ofrece }\end{array}$} & $\begin{array}{l}\text { Presenta res } \\
\text { mes, datos de } \\
\text { a su página } \\
\text { presentación } \\
\text { al libro en } \\
\text { debate guiac } \\
\text { enlace a la cr } \\
\text { suscripción a } \\
\text { electrónico }\end{array}$ & $\begin{array}{l}\text { enas del libro del } \\
\text { a autor con enlace } \\
\text { personal, video de } \\
\text { de la obra, acceso } \\
\text { varios formatos, } \\
\text { do por preguntas, } \\
\text { ítica especializada, } \\
\text { noticias por correo }\end{array}$ & $\begin{array}{l}\text { Selec } \\
\text { mes } r \\
\text { votos } \\
\text { recon } \\
\text { come } \\
\text { reseñ } \\
\text { autor } \\
\text { de la } \\
\text { privac } \\
\text { miem } \\
\text { géner } \\
\text { etc. }\end{array}$ & $\begin{array}{l}\text { ción de la lectura del } \\
\text { mediante sistema de } \\
\text { nendaciones de lectura, } \\
\text { entar o puntear libros, } \\
\text { ias de libros, datos del } \\
\text {, pequeños fragmentos } \\
\text { obra, enviar mensajes } \\
\text { dos y datos de cada } \\
\text { abro: libros, autores y } \\
\text { ros favoritos, amigos, }\end{array}$ & $\begin{array}{l}\text { Recibir por correo extracto de libros, } \\
\text { compartir lecturas y opiniones, recibir } \\
\text { notificaciones por correo electrónico, } \\
\text { intercambiar con el autor, publicación } \\
\text { de entrevistas y fotos de autores. }\end{array}$ \\
\hline
\end{tabular}

Fuente: Elaboración propia (2014).

Nota: EE.UU: Estados Unidos; RSS: Really Simple Sindication. 
- Diagnosticar los gustos, motivaciones y el comportamiento de los lectores.

- Conformar el grupo de usuarios.

- Definir los objetivos del club y las políticas del servicio.

- Evaluar las capacidades tecnológicas existentes, tanto desde el punto de vista del usuario, como de la biblioteca.

- Crear la página o grupo en la Web social.

- Elaborar los contenidos para el servicio: digitalizar portadas de los libros y capítulos del mismo, confeccionar reseña del título, biografía del autor, ofrecer enlaces a la crítica especializada.

- Proponer títulos de libros para que mediante sistema de votación los miembros decidan qué van a leer.

- Guiar e incentivar el debate entre los lectores, mediante preguntas.

- Supervisar y alertar ante incidencias, a los usuarios infractores de la Política del Servicio.

- Promocionar las actividades del club tradicional y otras actividades de la biblioteca.

- Evaluar continuamente el servicio y tramitar las sugerencias de los usuarios.

El primer elemento a considerar en la planificación del servicio, es conocer los intereses y necesidades de los lectores. Con esta información se procederá a conformar el Club de Lectura con los lectores que presentan similar interés por determinada temática, posteriormente con el consenso de todos los miembros se deberá definir los objetivos del club, las políticas del servicio y las estrategias a desarrollar para su difusión, pues algunos lectores en ocasiones prefieren recibir asesoría en lugar de actividades participativas. Las vías para desarrollar esta actividad pueden ser muy variadas, desde la observación del medio ambiente, la investigación, hasta el contacto personal con los lectores mediante entrevistas o encuestas.

En relación a las políticas del servicio, estas en lo fundamental, deben responder a cuatro dimensiones: las relativas a la organización del servicio, al comportamiento del personal que atenderá el mismo, a los deberes y derechos de los lectores y sobre lo concerniente al respecto a la legislación sobre Derecho de autor.
Antes de proceder a diseñar e implementar el servicio, se requiere de una evaluación de la infraestructura tecnológica con la que cuenta la organización y el usuario. A partir de esa valoración, se han de analizar las disímiles tecnologías existentes y ajustándose al estudio anterior, seleccionar la más idónea, que entre las funcionalidades más atractivas para los usuarios debe considerar: la capacidad para ofrecer facilidades para, de manera opcional, recibir notificación por correo electrónico, servir de espacio para proporcionar contacto con autores, preferentemente por chat, foro y otros servicios que potencien la participación activa de la comunidad usuaria.

En este punto resulta igualmente importante evaluar otros servicios similares y tomar de estos las mejores experiencias. Empezar el proceso de formar un club de lectura, investigando sobre el funcionamiento de clubes ya existentes, rinde buenos frutos en cuanto a mejorar el funcionamiento del mismo, logrando así minimizar los aspectos negativos, que ya previamente estas instituciones han identificado.

Antes de poner en marcha el Club de Lectura, en el medio tecnológico definido, un aspecto de primordial importancia es establecer los criterios para la selección de los libros, acción que se realizará en conjunto con algunos lectores seleccionados. Establecidos estos criterios, se determina el título a promover, se digitaliza su portada y se elabora una información detallada de dicha obra, incluyendo datos del autor, si existe la versión en digital de la obra, se deberán realizar todas las gestiones necesarias para obtener la licencia correspondiente y permitirles a los lectores poder leer en pantalla o descargarse la obra para un dispositivo de lectura. Un ejemplo de buenas prácticas en este sentido es el Club de Lectura Virtual de la Biblioteca de Barcelona $<$ http://www.clubdelectura.net> quien junto a la propuesta de título, presenta materiales de ayuda para la lectura, videos con entrevistas al autor o algunas escenas de la obra, enlaces a la crítica especializada, acceso a capítulos del libro, datos del autory de sus obras, así como información sobre el libro y los personajes.

Para determinar los títulos a promover, es recomendable elaborar una lista de al menos cinco opciones, para que mediante sistema de votación los miembros del club, determinen su favorito. Esta práctica es seguida por algunos de los servicios analizados, entre 
ellos los clubes de lectura de la Toronto Public Library $<$ http://www.torontopubliclibrary.ca/bookbuzz/> y de la Haliburton County Public Library's <http://www. goodreads.com/group/show/82817-hcpl-s-online-bookclub> y por Quelibroleo.com <http://www.quelibroleo. $\mathrm{com} />$. Resulta oportuno señalar que esta acción debe ser intencionada por el moderador del club, quien está apoyado en listas de best seller, folletos de editorial o reseñas de libros de otros clubes de lectura existentes en la red, y facilite información complementaria al grupo para ayudar la toma de decisión.

Una excelente vía para incentivar el debate es preparar una guía de lectura y en caso de no tener una disponible o de tiempo para su confección, se debe recurrir a elaborar preguntas o comentarios generales que ayuden a promover el debate. Alternativas para ello son: visitar otros clubes de lectura o sitios de promoción de libros, los cuales suelen proporcionar una breve descripción del mismo junto con comentarios y preguntas de los lectores, hacer que cada miembro, antes de comenzar la sección, anote una pregunta que luego es socializada o presentar fragmentos interesantes de la obra para mantener un hilo de conversación entorno al mismo.

En sentido general, las preguntas pueden estar relacionadas con: el valor de la obra, sobre los temas que más enfatiza el escritor y el aumento del interés del lector sobre los mismos, la evolución de los personajes en la historia y la identificación del lector con estos y la manera en que el escritor revela su visión del mundo a través de la obra y su incidencia en un cambio de actitud del lector ante la temática que se aborda o la asimilación de nuevo conocimiento que modifique algún aspecto de su vida, no descubierto con anterioridad. Los clubes de lectura de las Bibliotecas Municipales de A Coruña <http:// ciberclublectura.wordpress.com/> y de la Toronto Public Library <http://www.torontopubliclibrary.ca/bookbuzz/> incentivan la participación de sus lectores a través de las prácticas descritas anteriormente.

Para mantener una buena dinámica dentro del club de lectura virtual, el moderador o facilitador además de guiar el debate en torno a la obra del autor y al título que se presenta, debe animar la participación de los lectores, dar respuesta a los mensajes de los miembros, reseñar los libros y la información bibliográfica correspondiente, debe aplicar o hacer cumplir las reglas del servicio. Funciones que requieren que el moderador sea flexible, dedicado y con mesura, sobre todo para mantener una conversación equilibrada y el buen tono del debate.

Evidentemente la promoción del club de lectura es esencial para atraer a nuevos miembros, recomendando como vías: los concursos, forma divertida y gratificante de estimular la participación; unirse a otros clubes de lectura virtual; vincular el sitio del club con los de los autores que se promueven y con la biblioteca. Además no se deben olvidar las formas tradicionales, como la creación de folletos, poster y la promoción directa en actividades relacionadas con el libro. De los clubes analizados son ejemplo de incentivar la participación de sus lectores mediante variadas alternativas, el de la Toronto Public Library <http:// www.toronto publiclibrary.ca/bookbuzz/>y los portales BookCountry <http://bookcountry.com/> y Folec <http:// www.fomentolectura.es/>.

Porúltimo, es recomendableestablecer mecanismos de retroalimentación que permitan evaluar periódicamente el estado del proyecto y tomar medidas correctoras, modificar los objetivos o lo que sea necesario, mantener una actualización constate, así como, tramitar las sugerencias de los usuarios.

En resumen, el éxito del Club de Lectura Virtual no solo dependerá de una buena selección de las obras literarias a promover, más bien estribará en una actitud de escucha permanente de las aportaciones de todos los lectores, y de estimularlos y agradecerles como con su trabajo han contribuido al desarrollo de los hábitos de la lectura en la comunidad.

\section{Conclusión}

Innegable son los esfuerzos que se realizan por lograr que la lectura sea valorada por la sociedad y particularmente por las generaciones más jóvenes, en este sentido, la biblioteca para desarrollar acciones que contribuyan a este fin, primeramente ha de reconocer las tendencias actuales en el comportamiento de los lectores, reconociendo que estos se pueden tipificar, en cuanto al formato que prefieren para la lectura, en tradicionales, polivalentes y digitales. Por otro lado reconocer que en la actualidad, la lectura compite con una variedad de opciones donde pasar el tiempo libre y 
ratos de ocio, propiciadas en gran medida por el desarrollo de tecnologías más colaborativas y enfocadas a las expectativas de los usuarios.

En este sentido, propiciar un acercamiento del lector a los libros que le pueden ser de interés, requerirá aprovechar las potencialidades que diferentes tecnologías sociales brindan, espacios donde obtener información y compartir opiniones sobre libros y autores es una opción más. Particularmente se han documentado las oportunidades que brindan herramientas como los blog, wikis, sitios de redes sociales y diferentes plataformas para el intercambio de archivos audiovisuales y datos, tal como: YouTube, Flickr, Slideshare, RSS.

Al hacer una mirada a la web, múltiples son las iniciativas que se pueden valorar para dar los primeros pasos en el desarrollo e implementación de las actividades para el fomento de la lectura. Cada experiencia reseñada en este estudio permitió obtener información de primera mano y adquirir ideas para el desarrollo de estas actividades, con las cuales desde la biblioteca, en crear espacios que favorezcan el intercambio de opiniones y experiencias, que involucre y estimule la participación de los lectores e intente motivarlos a formar en ellos la necesidad de leer, como una opción de ocio.

Finalmente, a modo de recomendación, si se quiere ofrecer un servicio de promoción de la lectura, que resulte atractivo a los lectores, se ha de considerar como un elemento fundamental la creación de las condiciones para estimular la colaboración de los mismos (participación activa en el diseño, implementación, desarrollo y evaluación), resultando las tecnologías sociales un mecanismo idóneo para lograr cumplimentar estas acciones, logrando así que el club de lectura de la biblioteca se convierta en una comunidad de lectores colaborativa y participativa.

\section{Referencias}

Abello, L. Uso de Tic's en fomento a la lectura: experiencia en la biblioteca pública municipal de Doñihue y otras bibliotecas. Encuentro Nacional de BiblioRedes, 7., 2010, Olmué, Chile:Anales electrónicos... Olmué: Word Press, 2010. Disponible en: <http:// www.slideshare.net/BiblioRedes/>. Acceso en: 22 feb. 2013.

Abram, S. Promoting reading using this 2.0 Stuff. MultiMedia \& Internet@Schools, v.15,n.5, p.21-23, 2008.

Arreguin, C. Wikis. In: Hoffman, B. (Ed.). Encyclopedia of educational technology. 2004. Disponible en: <http:// www.etc.edu.cn/eet/eet/articles/wikis/index.htm>. Acceso en: 22 feb. 2013.

Arya, H.B.; Mishra, J.K. Oh! web 2.0, virtual reference service 2.0, tools \& techniques (II). Journal of Library \& Information Services in Distance Learning, v.6, n.1, p.28-46, 2012.

Bolan, K.; Canada, M.; Cullin, R. Web, library, and teen service 2.0. Young Adult Library Service v.5, n.2, p.40-43, 2007.

Buigues-García, M.; Giménez-Chornet, V. Impact of web 2.0 on national libraries. International Journal of Information Management, v.32, n.1, p.3-10, 2012.

Celaya, J. Fomento de la lectura en red. 2007. Disponible en: $<$ http://www.dosdoce.com/articulo/opinion/2832/fomentode-la-lectura-en-red/>. Acceso en: 22 feb. 2013.

Celaya, J. Las redes sociales en el fomento de la lectura en bachillerato. 2009. Disponible en: <http://www.plec.es/ documentos.php?id_seccion=6\&id_documento=155\&nivel= Bachillerato>. Acceso en: 22 feb. 2013.

Chawner, B.; Lewis, P. Wikiwikiwebs: New ways of communicating in a web environment. Information Technology and Libraries, v.25, n. 1, p.33-43, 2006.
Cruz González-Cutre, I.; Saurin Parra, J. Un viaje virtual por los clubes delectura.2008. Disponible en: <http://eprints.rclis.org/ bitstream/10760/12561/1/Art._Blogs-De_la_Cruz\%26Saurin. pdf>. Acceso en: 22 feb. 2013.

Hernandez Sampieri, R.; Fernandez Collado, C.; Baptista Lucio, P. Metodología de la investigación. 4.ed. México: McGraw Hill, 2006.

Lozano, R. El fomento de la lectura en la biblioteca pública 2.0: una apuesta por la innovación y el riesgo. Anuário ThinkEPI, v.4, p.87-90, 2010. Disponible en: <http://www.thinkepi.net/ el-fomento-de-la-lectura-en-la-biblioteca-publica-20-unaapuesta-por-la-innovacion-y-el-riesgo>. Acceso en: 22 feb. 2013.

Luo, L. Web 2.0 Integration in Information Literacy Instruction: An overview. The Journal of Academic Librarianship, v.36, n.1, p.32-40, 2009.

Manso-Rodríguez, R.A. Bibliotecas, fomento de la lectura y redes sociales: convirtamos amigos en lectores. El profesional de la información, v.21, n.4, p.401-405, 2012.

Reitz, J.M. ODLIS: online dictionary for library and information science, 2010. Available from: <http://www.abc-clio.com/ ODLIS/about.aspx>. Cited en: Feb. 222013.

Richardson, W. Blogs, wikis, podcasts, and other powerful web tools for classrooms. California: Corwin Press, 2006.

Seoane, C. Aplicaciones web 2.0 para fomentar la lectura. 2010. Disponible en: <http://www.slideshare.net/catuxa>. Acceso en: 22 feb. 2013. 
